\title{
GAUSSIAN PROCESS AND COMPBINED KERNEL SUPPORTED ANALYZING HYPER SUPERNATURAL REFLECTIVITY
}

\section{Sunil Singarapu ${ }^{1}$, Dr. Avinash Gour ${ }^{2}$}

${ }^{1}$ Research Scholar, Dept. of Electronics and Communication Engineering, Sri Satya Sai University of Technology \& Medical Sciences, Sehore, Bhopal-Indore Road, Madhya Pradesh, India.

${ }^{2}$ Research Guide, Dept. of Electronics and Communication Engineering, Sri Satya Sai University of Technology \& Medical Sciences, Sehore, Bhopal Indore Road, Madhya Pradesh, India.

Email: sunil.sun0505@gmail.com

Keywords:

SVM, Kernel, Gaussian Process, Reflection, ARMTO.

\begin{abstract}
ABSTRA C T
In this system, we gauge join a section choice technique, taking into account molecule swarm update (PSO), with a piece procedure, uphold vector machines (SVM), to lessen the dimensionality of hyper ridiculous information for demand. We review several undeniable kernals, including some improved for hyper absurd assessment. Specifically, an advancing segment called perception point subordinate (OAD) piece, from the outset pronounced for Gaussian Process descend into sin, was released up for SVM gathering. The SVM with the adjusted kernal was then applied to prompt the segment confirmation of a twofold kind of PSO. We embrace the strategy utilizing hyper powerful edifying records got of most recent tests from Western Australia. The test outcomes show that our framework can reasonably reduce the measure of highlights while keeping, or in any case, changing, the presentation of the SVM classifier. With current and moving toward symbolism spectrometers, mechanized band examination procedures are depended upon to draw in gainful unmistakable check of most significant social occasions to help improved treatment of terrible information into evaluations of biophysical factors.
\end{abstract}

Citation: Sunil Singarapu, Dr. Avinash Gour (2020). Gaussian Process And Compbined Kernel Supported Analyzing Hyper Supernatural Reflectivity. International Journal of Advanced Multidisciplinary Scientific Research (IJAMSR) ISSN:2581-4281, 2 (8), August 2019, pp $20-43$ 
IJAMSR 2 (8) www.ijamsr.com CrossRef: https://doi.org/10.31426/ijamsr.2019.2.8.1813

\section{Introduction}

In the inaccessible distinguishing composing, many coordinated and solo procedures have been delivered for multi-and hyper phantom picture portrayal (for instance most extraordinary likelihood classifiers, neural systems, euro-fleecy models, etc. In any case, a noteworthy issue concerning hyper ghastly data is the high number of ghostly gatherings and reasonably low number of named planning tests, which speaks to the outstanding Hughes wonder. This issue is ordinarily reduced by introducing a component decision/extraction step already prepartuation subordinate, and to a great extent requires from the previous data. Starting late, piece systems, for instance, reinforce vector machines (SVMs) or kernal Fisher separate examination, have shown radiant execution in hyper phantom data portrayal to the extent ing the hyper ghostly reduction of data dimensionally. Regardless, including such a phase is dreary, siexactness and healthiness. The characteristics of piece strategies make them suitable to deal with the issue of hyper ghastly picture gathering since they can manage tremendous data spaces capably, work with a for the most part low number of named planning tests, and oversee noisy examples in a fiery way. In any case, to the makers' data, kernal methodologies have so far thought about the ghostly information to develop the classifier and thusly, the spacial irregularity of the ghastly imprint has not been thought of. In this thesis, we unequivocally plan a full gathering of part supported classifiers that simultaneously think about spacial, supernatural, and close by crossinformation in a hyper ghastly picture. Thus, we abuse two especially fascinating characteristics of kernal procedures:

\section{Complex Kernels For Hyper Supernatural Image Classification.}

A full gathering of complex pieces for the mix of ghostly and intelligent information is presented here. Thus, three phases are followed:

1) Definition of pel. A pel substance $\mathbf{x}_{i}$ is re-imagined at the same time both in the supernatural domain utilizing its supernatural content, $\mathbf{x}_{i}^{\omega} \in \mathbb{R}^{N_{\omega}}$, also, in the spacial space by applying some component extraction to its encompassing region, $\mathbf{x}_{i}^{s} \in \mathbb{R}^{N_{s}}$, 
which yields Ns spacial (relevant) highlights.

2) Kernel calculation. When the spacial and supernatural element vectors $\mathbf{x}_{i}^{s}$ and $\mathbf{x}_{i}^{\omega}$ are built, distinctive kernal grids can be handily figured utilizing any reasonable portion work that satisfies Mercer's conditions.

3) Kernel blends. Now, we exploit the immediate aggregate of Hilbert Spaces by which (in any event two) Hilbert spaces Hk can merge into a greater Hilbert space. In the going with, we present four differing piece perspective for the joint thought of otherworldly and textural data in a united framework for hyper ghastly picture grouping.

\section{The Sonsy Features Approach}

The most normally got philosophy in hyper phantom picture gathering is to abuse the ghastly substance of a pel, $\mathbf{x}_{i} \equiv \mathbf{x}_{i}^{\omega}$. Be that as it may, execution can be improved by including both textural and supernatural data in the classifier. Note that if the picked planning $\varphi$ is a difference in the connection $\mathbf{x}_{i} \equiv\left\{\mathbf{x}_{i}^{s}, \mathbf{x}_{i}^{\omega}\right\}$, at that point the contrasting 'sonsy' piece structure is:

$$
K_{\{s, \omega\}} \equiv K\left(\mathbf{x}_{i}, \mathbf{x}_{j}\right)=\left\langle\phi\left(\mathbf{x}_{i}\right), \phi\left(\mathbf{x}_{j}\right)\right\rangle,
$$

Which rejects unequivocal cross relations

$$
\begin{aligned}
& \text { between } \mathbf{x}_{i \text { and }}^{s} \mathbf{x}_{i}^{\omega} \\
& \phi\left(\mathbf{x}_{i}\right)=\left\{\varphi_{1}\left(\mathbf{x}_{i}^{s}\right), \varphi_{2}\left(\mathbf{x}_{i}^{\omega}\right)\right\}
\end{aligned}
$$

Also, the comparing spot item can be effectively registered as follows:

$$
\begin{aligned}
& K\left(\mathbf{x}_{i}, \mathbf{x}_{j}\right)=\left\langle\boldsymbol{\phi}\left(\mathbf{x}_{i}\right), \boldsymbol{\phi}\left(\mathbf{x}_{j}\right)\right\rangle \\
& =\left\langle\left\{\varphi_{1}\left(\mathbf{x}_{i}^{s}\right), \varphi_{2}\left(\mathbf{x}_{i}^{\omega}\right)\right\},\left\{\varphi_{1}\left(\mathbf{x}_{j}^{s}\right), \varphi_{2}\left(\mathbf{x}_{j}^{\omega}\right)\right\}\right\rangle \\
& =K_{s}\left(\mathbf{x}_{i}^{s}, \mathbf{x}_{j}^{s}\right)+K_{\omega}\left(\mathbf{x}_{i}^{\omega}, \mathbf{x}_{j}^{\omega}\right)
\end{aligned}
$$

\section{The Straightness Summing Up Kernel}

A clear complex piece solidifying unearthly and textural information typically starts from the connection of nonlinear changes of $\mathbf{x}_{i}^{s}$ and $\mathbf{x}_{i}^{\omega}$. Let us assume two nonlinear changes $\varphi_{1}(\cdot)$ and $\varphi_{2}(\cdot)$ into Hilbert spaces $\mathrm{H}_{1}$ and $\mathrm{H}_{2}$, respectively. At that point, the accompanying change can be built: 
IJAMSR 2 (8) www.ijamsr.com CrossRef: https://doi.org/10.31426/ijamsr.2019.2.8.1813

Point that the plan is conveyed as the total of positive clear systems speaking to the textural and phantom accomplices, selfrulingly Note that diminish $\left(\mathbf{x}_{i}^{\omega}\right)=N_{\omega}, \operatorname{dim}\left(\mathbf{x}_{i}^{s}\right)=$ $N_{s}$, and $\operatorname{dim}(K)=\operatorname{dim}\left(K_{s}\right)=\operatorname{dim}\left(K_{\omega}\right)=n \times$

\section{The Weighted Summing Up Kernel}

By misusing Property (2) in Proposition 1, a complex part that adjusts the spacial and supernatural substance in (10) can likewise be made, as follows:

\section{$K\left(\mathbf{x}_{i}, \mathbf{x}_{j}\right)=\mu K_{s}\left(\mathbf{x}_{i}^{*}, \mathbf{x}_{j}^{*}\right)+(1-\mu) K_{\omega}\left(\mathbf{x}_{i}^{\omega}, \mathbf{x}_{j}^{\omega}\right)$}

Where $\mu$ is a positive certifiable regarded free limit $(0<\mu<1)$, which is tuned in the preparation measure and builds up a tradeoff between the special and ghastly information to organize a given pel

\section{The Cross-Data Kernel}

The past piece classifiers can be supportively changed to speak to the cross association between the otherworldly and spacial information. Acknowledge a nonlinear planning $\phi(\bullet)$ to a Hilbert space $\mathrm{H}$ and three direct changes Ak from $\mathrm{H}$ to $\mathrm{Hk}$, for $\mathrm{k}=1,2,3$. Let us build up the going with complex vector:
$\phi\left(\mathbf{x}_{i}\right)=\left\{\mathbf{A}_{1} \varphi\left(\mathbf{x}_{i}^{*}\right), \mathbf{A}_{2} \varphi\left(\mathbf{x}_{i}^{\psi}\right), \mathbf{A}_{3}\left(\varphi\left(\mathbf{x}_{i}^{*}\right)+\varphi\left(\mathbf{x}_{i}^{-}\right)\right)\right\}$

Furthermore, register the dot multiplication,

$$
\begin{aligned}
& K\left(\mathbf{x}_{i}, \mathbf{x}_{j}\right)=\left\langle\phi\left(\mathbf{x}_{i}\right), \phi\left(\mathbf{x}_{j}\right)\right\rangle \\
& =\phi\left(\mathbf{x}_{i}^{s}\right)^{\top} \mathbf{R}_{1} \phi\left(\mathbf{x}_{j}^{s}\right)+\phi\left(\mathbf{x}_{i}^{\omega}\right)^{\top} \mathbf{R}_{2} \phi\left(\mathbf{x}_{j}^{\omega}\right)
\end{aligned}
$$$$
+\phi\left(\mathbf{x}_{i}^{s}\right)^{\top} \mathbf{R}_{3} \phi\left(\mathbf{x}_{j}^{\omega}\right)+\phi\left(\mathbf{x}_{i}^{\omega}\right)^{\top} \mathbf{R}_{3} \phi\left(\mathbf{x}_{j}^{s}\right)
$$

Where

$\mathbf{R}_{1}=\mathbf{A}_{1}^{\top} \mathbf{A}_{1}+\mathbf{A}_{3}^{\top} \mathbf{A}_{3}, \mathbf{R}_{2}=\mathbf{A}_{2}^{\top} \mathbf{A}_{2}+\mathbf{A}_{3}^{\top} \mathbf{A}_{3}$,

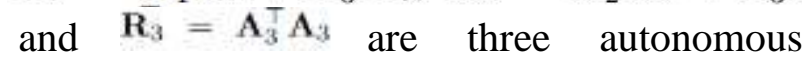
positive distinct lattices. Additionally to the immediate summing up piece, it very well may be shown that (12) can be communicated as the total of positive distinct grids, representing the supernatural, textural,and cross-terms among textural and unearthly accomplices:

$$
\begin{aligned}
& K\left(\mathbf{x}_{i}, \mathbf{x}_{j}\right)=K_{s}\left(\mathbf{x}_{i}^{s}, \mathbf{x}_{j}^{s}\right)+K_{\omega}\left(\mathbf{x}_{i}^{\omega}, \mathbf{x}_{j}^{\omega}\right) \\
& +K_{s \omega}\left(\mathbf{x}_{i}^{s}, \mathbf{x}_{j}^{\omega}\right)+K_{\omega s}\left(\mathbf{x}_{i}^{\omega}, \mathbf{x}_{j}^{s}\right)
\end{aligned}
$$

The primary constraint for this intend to be considerable is that $\mathbf{x}_{i}^{s}$ and $\mathbf{x}_{j}^{\omega}$. need to have a similar measurement $\left(N_{\omega}=N_{s}\right)$. A natural 
IJAMSR 2 (8) www.ijamsr.com CrossRef: https://doi.org/10.31426/ijamsr.2019.2.8.1813

case of this complex piece would be according to the accompanying. Let the spacial highlights $\mathbf{X}_{i}^{s}$ be the normal of the reflectivity esteems in a given window around pel $\mathrm{x}_{\mathrm{i}}$ for each band, and let the supernatural features $\mathbf{x}_{j}^{\omega}$. be the genuine ghastly signature $\left(\mathbf{x}_{i}=\mathbf{x}_{i}^{\omega}\right)$. Then, $K_{s}\left(K_{\omega}\right)$ represent the separation grid among

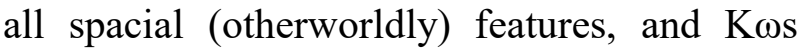
addresses the closeness arrange confined by the partitions among the spectra and the showed up at the midpoint of neighborhoods. Note that dealing with the minimization issue in a wide range of complex parts requires indistinguishable number of imperatives from in the regular SVM calculation, and in this way no extra computational endeavors are initiated in the introduced approaches.

\section{Supernatural Band Selection For} Aggregation Characteristics Rescue Using Gaussian Processes Regression

Estimation, relapse and capacity guess are old, to a great extent considered issues in measurements and AI. The difficult comes down to streamline a misfortune (cost, vitality) work over a class of capacities. An enormous class of relapse issues specifically is characterized as the joint minimization of a misfortune work representing mistakes of $f \in \mathcal{H}$ to be learned, and a regularization term, $\Omega\left(\|f\|_{\mathcal{H}}^{2}\right)$ that controls its ability (overabundance of adaptability). The issue can be drawn closer inside a Bayesian nonparametric structure, and a few calculations are accessible, for example, the pertinence vector machine or Gaussian Processes relapse (GPR) in which we will center here. GPR is comparable in nature to portion edge relapse (otherwise known as least square help vector machine) and apparatus. Be that as it may, because of their high computational multifaceted nature they didn't turn out to be generally applied instruments in AI up to this point.

GPR can be deciphered as a group of portion strategies with the extra kernal of leeway of giving a full restrictive factual portrayal for the anticipated variable, which can be principally used to set up certainty stretches and to set hyper-boundaries. To put it plainly, GPR expect that a Gaussian process earlier administers the arrangement of conceivable dormant capacities (which are surreptitiously), and the probability (of the idle capacity) and 
IJAMSR 2 (8) www.ijamsr.com CrossRef: https://doi.org/10.31426/ijamsr.2019.2.8.1813

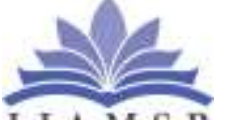

International Journal of

I J A M S R

Advanced Multidisciplinary Scientific Research (IJAMSR) ISSN:2581-4281

perceptions shape this preceding produce back probabilistic assessments. Therefore, the joint dispersion of preparing and test information is a multidimensional Gaussian and the anticipated circulation is evaluated by molding on the preparation information.

\section{Global affectability Analysis}

Remembering that GPR-BAT is a quantifiable, data driven strategy, it is grasped that the hugeness of the procured best performing bunches are by and large subject to the idea of the introduced dataset. Here, to check the criticalness of best performing gatherings, they will be contemplated against a change supported overall affectability examination (GSA) of the notable shade radioactive trade simulation PROSAIL (PROSPECT4 + SAIL).

ARTMO GPR-BAT module

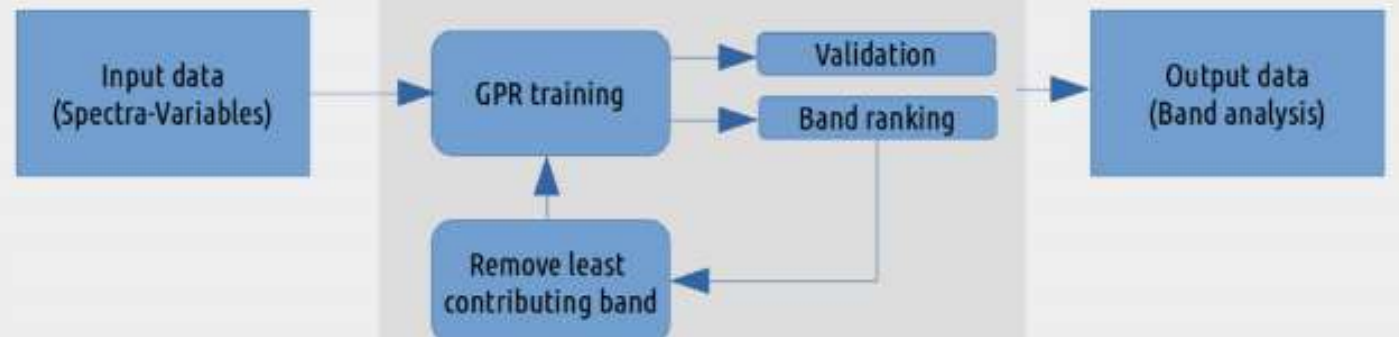

Figure 1: Schematic flow diagram of GPR-BAT within ARTMO's MLRA toolbox

The strategy can be used to recognize the most remarkable elements impacting simulation yields. In distinction supported GSA, the responsibility of every data variable to the assortment in yields is shown up at the midpoint of over the assortment of all data factors, i.e., all. 
IJAMSR 2 (8) www.ijamsr.com CrossRef: https://doi.org/10.31426/ijamsr.2019.2.8.1813

International Journal of Advanced Multidisciplinary Scientific Research (IJAMSR) ISSN:2581-4281

Table 1: Limits of used involve variables of the PROSAIL with a solar sphere angle of $30^{\circ}$

\begin{tabular}{|c|c|c|c|c|}
\hline \multicolumn{2}{|c|}{ Model variables } & Units & Minimum & Maximum \\
\hline \multicolumn{5}{|c|}{ Leaf variables: PROSPECT-4 } \\
\hline$N$ & Leaf structure index & Unit less & 1.2 & 2.6 \\
\hline LCC & Leaf chlorophyll content & {$\left[\mu \mathrm{g} / \mathrm{cm}^{2}\right]$} & 0 & 80 \\
\hline$C_{m}$ & Leaf dry matter content & {$\left[\mathrm{g} / \mathrm{cm}^{2}\right]$} & 0.001 & 0.02 \\
\hline LWC & Leaf water content & {$\left[\mathrm{g} / \mathrm{cm}^{2}\right]$} & 0.001 & 0.05 \\
\hline \multicolumn{5}{|c|}{ Canopy variables: SAIL } \\
\hline LAI & Leaf area index & {$\left[\mathrm{m}^{2} / \mathrm{m}^{2}\right]$} & 0 & 7 \\
\hline LAD & Leaf angle distribution & {$\left[{ }^{\circ}\right]$} & 30 & 60 \\
\hline
\end{tabular}

Info factors are changed together. Starting late a GSA instrument stash has been made inside the ARTMO pack, where the GSA assessment of RTMs embedded in ARTMO (for instance PROSAIL) has been generally robotized. The procedure for was realized, which has been shown to be fruitful in perceiving both the essential affectability impacts (first-demand impacts, i.e., the duty to the difference in the simulation yield by every data variables, $\mathrm{Si}$ ) and full scale affectability impacts (the essential solicitation impacts notwithstanding joint efforts with other data factors, STi) of data factors .

This made 9000 reenactments with directional reflectivity at yields some place in the scope of 400 and 2500 nmat $1 \mathrm{~nm}$ increments. Only hard and fast solicitation affectability impacts (STi) imparted as rates were suspected of.

\section{Features For Aggregation}

In this area, we present a conventional component extraction plot for administered grouping issues managing hyper supernatural information. Instead of a novel strategy, it depends on wavelet coefficients, consolidated to a proficient best in class include determination plot. We present another way to deal with aggregation inflection location, exploiting the significant information contained in the phantom mark of aggregation. Regardless, the presented feature extraction plan is nonexclusive, it may be said that it performs well, paying little psyche to the nature and multifaceted nature of the application and detector qualities (i.e., check and size of ghostly gatherings) Ad hoc features, for instance, limited band records are organized considering unequivocal applications and are indisputably not customary. They 
IJAMSR 2 (8) www.ijamsr.com CrossRef: https://doi.org/10.31426/ijamsr.2019.2.8.1813

similarly depend upon detector characteristics.

This is furthermore the circumstance if a foreordained number of phantom gatherings are chosen.

\section{Aggregation Files}

The clearest list of abilities is given by the reflectivity in each otherworldly band. The high association among them and the issue of high dimensional data, suggest concentrating just on a picked number of gatherings. Much of the time, a straight mix of gatherings or a normalized qualification of gatherings is held. Aggregation inflection can every so often be seen by methods for a move of the red edge toward smaller frequencies, similarly suggested as the blue move. Thusly, such records depicting the red-edge have been moved in the composition for perceiving aggregation inflection.

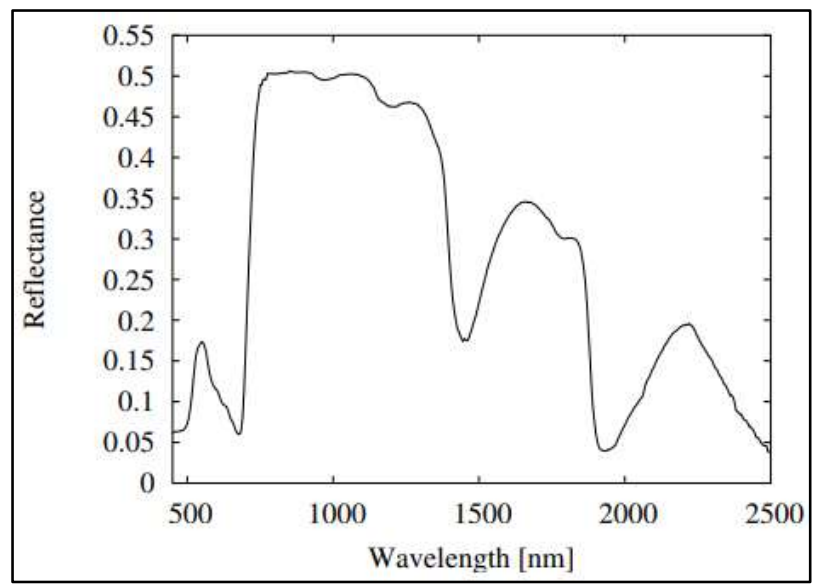

Figure 2: Example of a leaf reflectivity signature.

In Figure 2, we focus on scales contrasting with $\mathrm{j}=6$ and $\mathrm{j}=7$. The high pass channel identifying with the HAAR wavelet recognizes the serious edges in aggregation phantom imprint. Consideration that for this conversation, we just viewed as a solitary range On the off chance that we are keen on the distinctive conduct of two supernatural marks as in characterization different scales can turn out to be more significant. 
IJAMSR 2 (8) www.ijamsr.com CrossRef: https://doi.org/10.31426/ijamsr.2019.2.8.1813

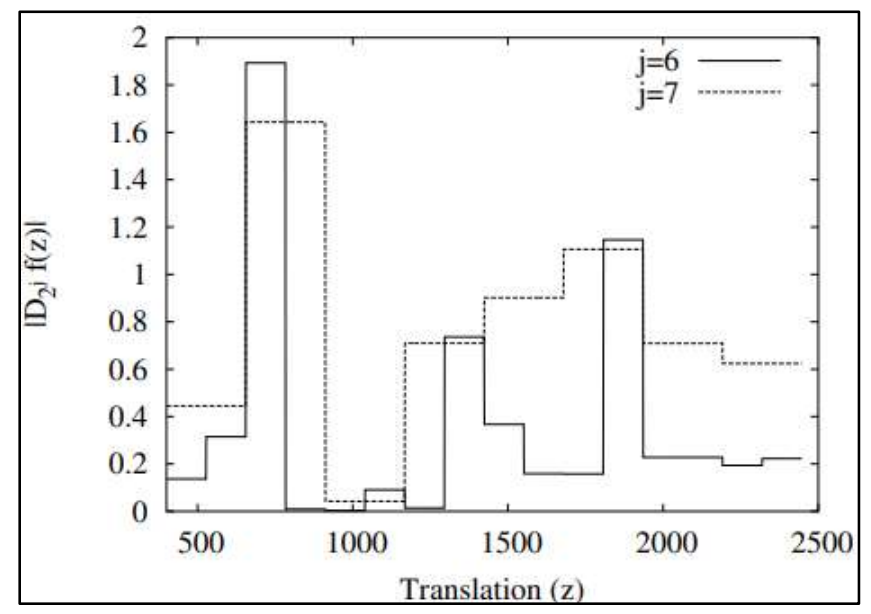

Figure 3: correct values of the discrete wavelet transform.

At each scale $\mathrm{j}$ by taking the root mean squared coefficients regards:

$$
E_{j}=\sqrt{\frac{1}{N_{j}} \sum_{x=0}^{N_{j}}\left[D_{2^{j}} f(x)\right]^{2}},
$$

Along these lines, the imperativeness ascends to the difference in the detail histogram. Using essentialness as a component depicts the detail histogram by a Gaussian. The essentialness features of the DWT for a comparable aggregation mark. In simultaneousness for figure 3.3 with the recognition made above, scale $\mathrm{j}=6$ is explicitly convincing, with an imperativeness content standing separated of the standard twist. 
IJAMSR 2 (8) www.ijamsr.com CrossRef: https://doi.org/10.31426/ijamsr.2019.2.8.1813

\section{Advanced Multidisciplinary Scientific Research (IJAMSR) ISSN:2581-4281}

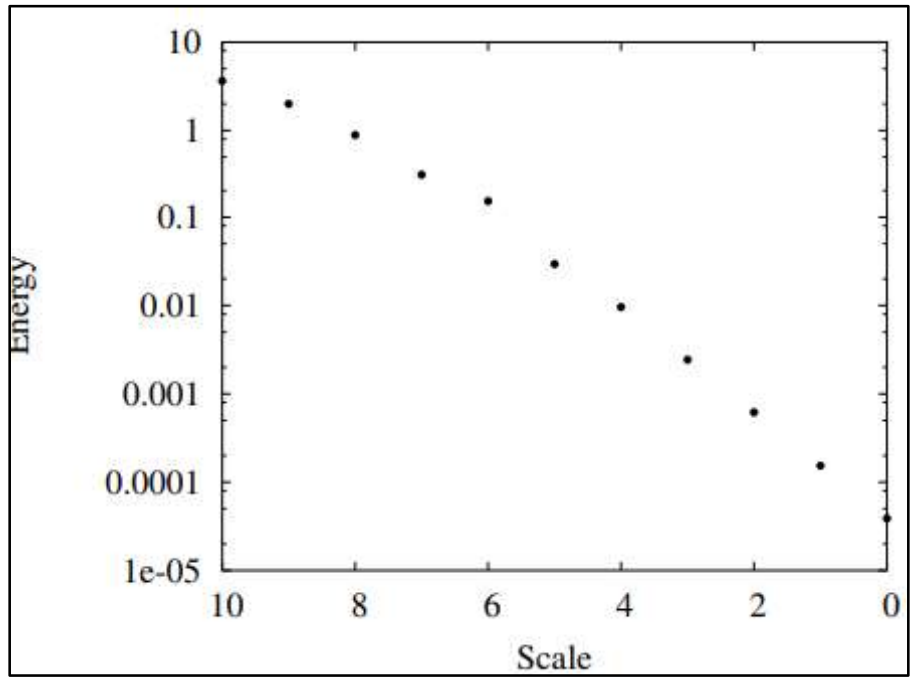

Figure 4: Energy Derived Discrete wavelets

In figure 3.4, be that as it might, these $\mathrm{n}$ features are not so much the ideal mix, i.e., including the resulting best single component to the best likely would exclude information for class separation.

\section{Sequential Floating Selection}

Plainly it requires just $\frac{(N+M)(N-M+1)}{2}$ assessments. Downside of this straightforward choice plan is that it can get stuck in a neighborhood least, since it can't right for recently included highlights. A refreshed interest procedure is moved by Pudil, evading the local least utilizing the consecutive coasting forward determination (SFFS.
Afterward advance, at least one in reverse progresses are taken, i.e., ousting an earlier picked variable to check whether the reparability compute can be extended at that level. The SFFS Figureuring can be depicted as in count 3.1: 
IJAMSR 2 (8) www.ijamsr.com CrossRef: https://doi.org/10.31426/ijamsr.2019.2.8.1813

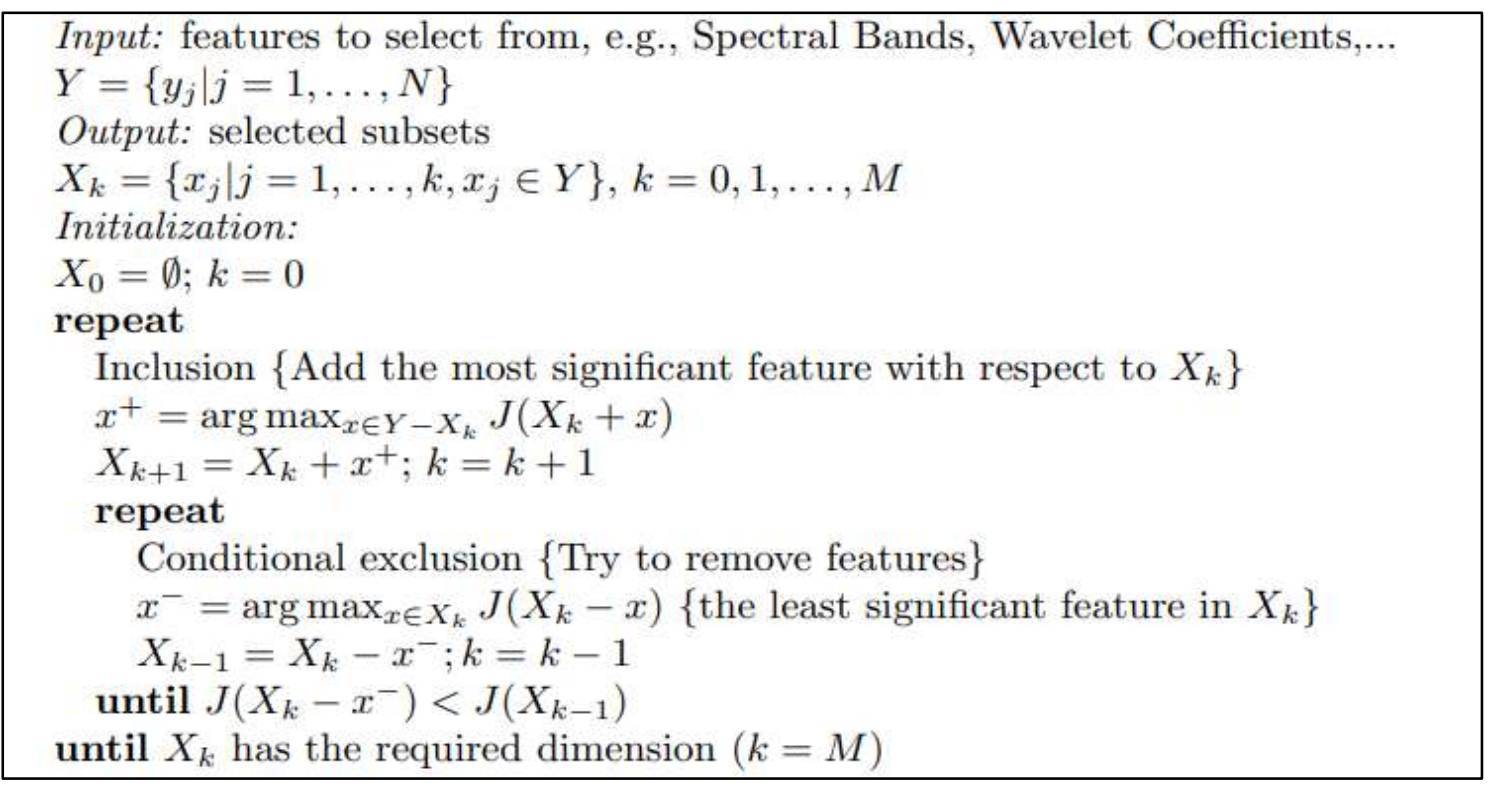

\section{Analysis On Inflection Sensing In Apple Orchards}

Hyper powerful far off distinguishing has expanded a lot of interest, also for cultivating applications. This is a remarkably upheld simulation that depicts the radioactive exchange inside the leaves by utilizing support and dissipating coefficients. It may be fomented with fitting it to the purposeful reflectivity spectra, by and large gained from specific leaves or little developing yields. This awards studying the chlorophyll; dry issue and water focus close by a leaf structure limit. This is the subject of part II of this theory. In any case, sign of weight should be acquired really from the got reflectivity expand. A few weight records have been proclaimed for pressure territory. They tons of social events, or then again attributes of the red-edge

Degrees are commonly taken care of from leaf reflectivity's that are evaluated in the assessment office utilizing a checking spectra radio meter with high spooky target. It isn't clear to get the summaries to mobile detectors with various focal points. The exactness at which the move in the red-edge position can be settled from mobile spectra is a lot of lower than from ground-upheld spectra. A red-edge 
IJAMSR 2 (8) www.ijamsr.com CrossRef: https://doi.org/10.31426/ijamsr.2019.2.8.1813

set up record doesn't just depend concerning the appalling, yet what's more on the Spacial Resolution of the finder. The particular significance of a weight archive extraordinarily relies on the particular idea of the current issue (unequivocal sort of total, inflection or possibly indicator). Getting a handle on a current record isn't ensured to work for the particular issue sufficiently close, and reasoning another summary requires express earlier information on the irksome that most probable won't be accessible. For this situation study, we rather followed a more standard way of thinking, by utilizing the reflectivity run without getting highlights from it from the earlier, as is finished with the radioactive exchange models or conglomeration inflection records. This technique has the extraordinary position that no past data of the particular collection inflection issue or obviously applied identifier is required.

This should be conceivable by applying all the accessible reflectivity social affairs of a range as highlights in a planned blueprint procedure. It is striking that the introduction of a classifier diminishes with higher number of highlights an irksome that is recognizable for hyper apparition information. Bruce announced diminishing the dimensionality of hyper spooky reflectivity spectra by a couple, say n, highlights passing on the best one-dimensional solicitation. Notwithstanding, these $n$ highlights are less the ideal mix, i.e., including the subsequent best single part to the best probably wo avoid data for class bundle.

Accordingly, we followed the methodology of portrayed in assessment 3.1. Each time an part is fused, by taking a gander at the best consolidated presentation, accordingly redesigning the multivariate class reparability. For this condition study, we separated the pronounced affirmation framework with the mix of the best single highlights for the issue of collection inflection territory.

The reflectivity esteems themselves probably won't be the most sensible portrayal. The craving to convey standard highlights, liberated from the apparition objective legitimizes the utilization of multi target delineations, for example, the ones produced using wavelet changes. Wavelet highlights have been utilized discontinuously for get-together purposes. Wavelets address the reflectivity run at various scales. 
IJAMSR 2 (8) www.ijamsr.com CrossRef: https://doi.org/10.31426/ijamsr.2019.2.8.1813

Highlights from express scales relate to unequivocal repulsive targets. The scales, from which highlights are picked, uncover data about material absurd target levels. This awards, for instance, looking at the essential apparition objective for the current application. Additionally, this point was investigated. To support the pronounced procedure, the affirmation of worry in trademark thing farms was thought of.

Foliar reflectivity appraisals were finished with an accommodating field spectra radio meter on the new leaves. Inflection records chose the leave spectra uncovered a huge kernal of the focused on tests, validating that the trees were in reality under pressure. All things considered, the standard framework made an overwhelming showing up, seeing all the focused on leaves from the reference leaves. The analyses were rehashed for different weight types and common thing assortments.

Models are demonstrated where the weight records can no longer see pressure, while the standard strategy despite everything can. For the use of pressure region in typical thing domains, the wavelet-upheld highlights are appeared to outsmart the unadulterated appalling reflectivity. Essentially, the methodology for drifting inquiry confirmation is appeared to beat picking the best single highlights.

At long last, the made sure about spectra are subsample, consequently mirroring a hyper appalling finder, to be utilized for conveying mobile and space borne information. It is demonstrated that the open apparition objective is acceptable for perceiving pressure.

\section{Inflection Sensing Using Aggregation Inflection}

Files Limits of the PROSPECT model, found by simulation reversal, were used for isolating among pressure besides, reference. The simulation boundaries were gotten by restricting the squared complexity between the simulation and assessed reflectivity go. For this examination, the structure limit was ideal to isolate among pressure what's more, reference, with $8 \%$ mess up rate. In the simulation this limit is liable for then again the reflectivity of the close infrared. Chlorophyll obsession apparently was not generally extraordinary for inflection and reference leaves. The normal decrease in chlorophyll obsession as a result of the nitrogen inadequacy didn't happen. 
IJAMSR 2 (8) www.ijamsr.com CrossRef: https://doi.org/10.31426/ijamsr.2019.2.8.1813

Appropriately, the move of the red-edge was missing and most of the related weight documents will without a doubt miss the mark. From all of the 18 weight records attempted, Rs lead to the best weight disclosure result.

\section{Inflection Arrangement Utilizing Supernatural Groups}

In the going with investigations, the display of the declared nonexclusive procedure was elucidated. In Figure 3.5, the request botches alongside the sureness extends are showed up in limit of the amounts of picked features from 1 to 9 . To dissect, the game plan botch using the most perfect aggregation inflection record Rs is moreover appeared.

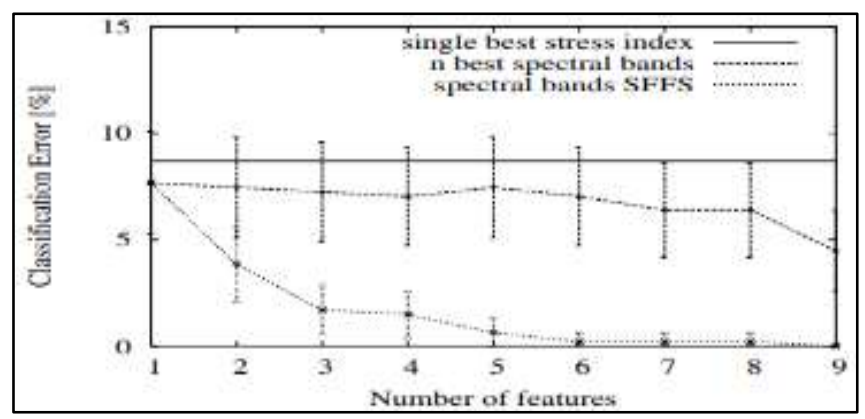

Figure 5: Characterization mistake for reflectivity highlights, chosen with $\mathbf{n}$ best (ran) and SFFS (specked), contrasted with the best intonation file Rs (strong)

From the Figure indisputably the use of the complete range improved outcomes, appeared differently in relation to the usage of a singular weight record. The more reflectivity features picked, the better the request result. Exactly when the unearthly features were picked autonomously, using the $\mathrm{n}$ best strategy, results didn't improve much with higher number of picked features.

\section{Inflection Order Utilizing Wavelet Features}

Again, the two component decision frameworks: $\mathrm{n}$ best and SFFS were contemplated. The outcomes are showed up in Figure. 3.6. The outcome for the most perfect aggregation inflection record is in like manner showed up. Results resemble the outcomes for the otherworldly features: applying $\mathrm{n}$ best assurance didn't improve results with higher number of picked highlights, while applying SFFS did. 


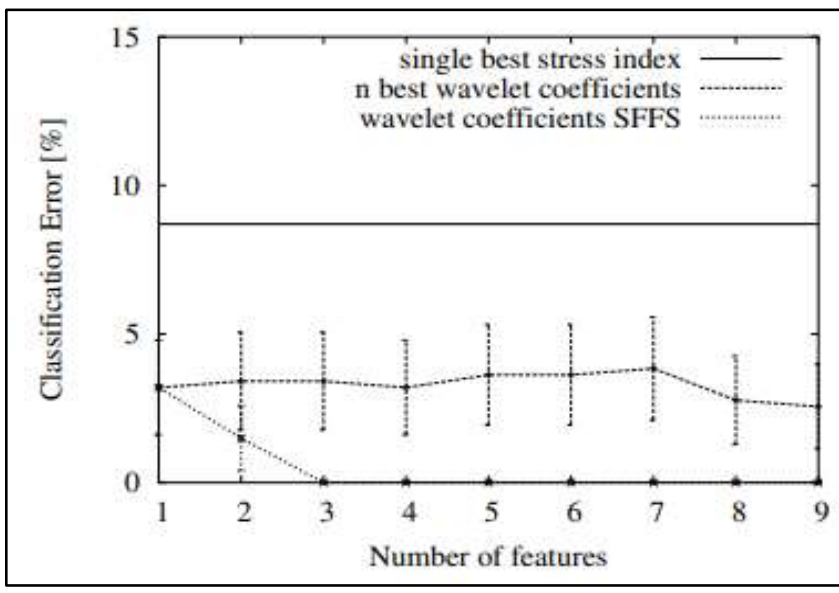

Figure 6: Arrangement mistake utilizing wavelet highlights, chosen with $\mathbf{n}$ best (ran) and SFFS (spotted), contrasted with the best emphasis file Rs (strong)

When differentiating Figure. 5 and Figure. 6, clearly the wavelet coefficients beat the reflectivity features. Differentiations are also showed up in a part of the aggregation inflection records, and are undeniably used in a customary multi objective framework.

\section{Simulation Development}

Investigations were finished using the common AVIRIS picture accepted authority over NW. We ousted 20 boisterous gatherings covering the locale of water osmosis, finally worked with 200 otherworldly gatherings. In every case, we used the polynomial kernal $(d=\{1, \ldots, 10\})$ for the supernatural highlights as indicated by past outcomes and utilized the $\operatorname{RBF}$ portion $\left(\sigma=\left\{10^{-1}, \ldots, 10^{3}\right\}\right)$ for the spacial features according to the territory presumption in the spacial space. By ethicalness of the weighted summing up piece, $\mu$ was vacillated in steps of 0.1 in the range $[0,1]$.

For straightforwardness what's more, for illustrative purposes, $\mu$ was the proportional for each named class in our assessments. For the 'sonsy' $\left(K_{\{s, \omega\}}\right)$ what's more, cross-data $\left(K_{B \omega}, K_{\omega s}\right)$ viewpoint, we utilized the polynomial piece. A one-against-one multi gathering plan was gotten in the two cases. The most fundamental yet historic spacial features xs I that can be taken out from a given region rely upon second norms. In this thesis, we consider the underlying two second to fabricate the spacial parts. 
IJAMSR 2 (8) www.ijamsr.com CrossRef: https://doi.org/10.31426/ijamsr.2019.2.8.1813

International Journal of Advanced Multidisciplinary Scientific Research (IJAMSR) ISSN:2581-4281

Table 2: by and large precision, oa[\%], and kappa measurement, $\kappa$, on the approval sets

\begin{tabular}{|c|c|c|c|c|}
\hline & \multicolumn{2}{|c|}{$\begin{array}{l}\text { SUBSET } \\
\text { SCENE }\end{array}$} & \multicolumn{2}{|c|}{$\begin{array}{l}\text { WHOLE } \\
\text { SCENE }\end{array}$} \\
\hline & $\mathbf{O A}[\%]$ & $\kappa$ & $\mathbf{O A}[\%]$ & $\kappa$ \\
\hline \multicolumn{5}{|l|}{$\overline{\text { Spectral classifiers }}^{\dagger}$} \\
\hline Euclidean [15] & 67.43 & - & 48.23 & - \\
\hline bLOOC+DAFE+ECHO [15] & 93.50 & - & 82.91 & - \\
\hline$K_{\omega}[7]$ & $\overline{95.90}$ & - & $\overline{87.30}$ & - \\
\hline$K_{\omega}$ developed in this paper & 95.10 & 0.94 & 88.55 & 0.87 \\
\hline \multicolumn{5}{|l|}{ Spatial-spectral classifiers } \\
\hline \multicolumn{5}{|l|}{ Mean } \\
\hline$K_{s}$ & 93.44 & 0.92 & $\underline{84.55}$ & 0.82 \\
\hline$K_{\{s, \omega\}}$ & 96.84 & 0.97 & $\overline{94.21}$ & 0.93 \\
\hline$K_{s}+K_{\omega}$ & 97.12 & 0.97 & 92.61 & 0.91 \\
\hline$\mu K_{s}+(1-\mu) K_{\omega}$ & 97.43 & 0.97 & 95.97 & 0.94 \\
\hline$K_{s}+K_{\omega}+K_{s \omega}+K_{\omega s}$ & 97.44 & 0.97 & 94.80 & 0.94 \\
\hline \multicolumn{5}{|l|}{ Mean and standard deviation ${ }^{7}$} \\
\hline$K_{s}$ & 94.86 & 0.94 & $\frac{88.00}{1.9}$ & 0.86 \\
\hline$K_{\{s ; \omega\}}$ & 98.23 & 0.97 & $\overline{94.21}$ & 0.93 \\
\hline$K_{s}+K_{\omega}$ & 98.26 & 0.98 & 95.45 & 0.95 \\
\hline$\mu K_{s}+(1-\mu) K_{\omega}$ & 98.86 & 0.98 & 96.53 & 0.96 \\
\hline
\end{tabular}

One stand out from the data and results uncovered in is that they inspected the scene using 17 classes (Soybeans-no till was part into two classes) however we used 16 classes. Moreover note that the usage of the LOOC calculation as opposed to the bLOOC calculation could improve execution, as declared in. Complexities between the got accuracy's uncovered in and the presented here could be a result of the self-assertive example assurance; in any case they are not genuinely basic. $\$$ Note that by using mean and standard deviation features, $N_{\omega} \neq N_{s}$ what's more, consequently no cross kernals $\left(K_{s \omega}\right.$ or $\left.K_{\omega s}\right)$ can be built.

\section{Simulation Comparison}

Table shows the endorsement aftereffects of a couple of classifiers for the two pictures. We consolidate results from six section classifiers: unearthly $(\mathrm{K} \omega)$, important $(\mathrm{Ks})$, the sonsy system $(\mathrm{K}\{\mathrm{s}, \omega\})$, and the three presented complex kernals. Additionally, two standard methods are consolidated for benchmark assessment: bLOOC + DAFE + ECHO, which use legitimate and unearthly information to amass homogeneous articles, and the Euclidean classifier, which just uses the ghastly information. All models are taken a gander at mathematically (all things considered precision, $\mathrm{OA}[\%]$ ) and genuinely (kappa test and Wilcox on rank sum test). 
IJAMSR 2 (8) www.ijamsr.com CrossRef: https://doi.org/10.31426/ijamsr.2019.2.8.1813

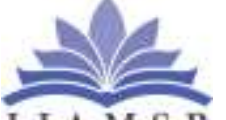

International Journal of

I J A M S R

Advanced Multidisciplinary Scientific Research (IJAMSR) ISSN:2581-4281

The outcomes found the center estimation of in excess of 10 sporadic recognize that were gotten the opportunity to avoid inclined finishes. Regardless, all piece supported methods duplicate better (and genuinely vital) portrayal results than past techniques (essential Euclidean and LOOC-supported procedure), as of late depicted in. It is also huge that the applicable kernal classifier Ks alone conveys extraordinary outcomes in the two pictures, essentially in light of the proximity of immense homogeneous classes and the high spacial Resolutionof the detector.

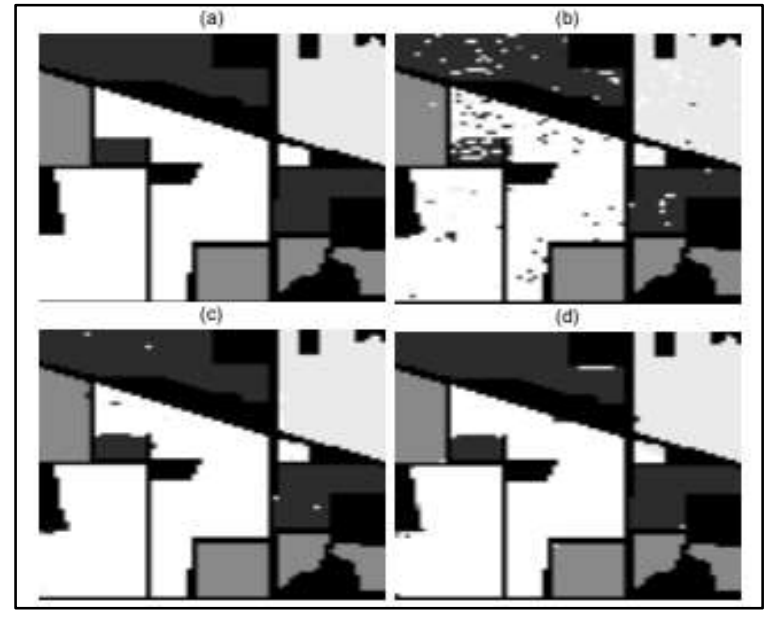

Figure 7: Characterization brings about the subset picture. (a) Labeled scene, and grouping maps utilizing the (b) logical piece, Ks (window size: $7 \times 7$ ), (c) powerful part, ${ }^{K_{\omega}}$, and (d) weighted summing up kernel $\left(\mu K_{s}+(1-\mu) K_{\omega}, \mu=0.2\right.$ window size: $\left.7 \times 7\right)$.

In any case, the precision is inferior contrasted with the great ghastly segment classifiers (both executed here and in which displays the relevance of the otherworldly information for hyper ghostly picture gathering. In addition, it justifies referring to that all complex piece classifiers improved the outcomes got by the common ghostly segment, which certifies the authenticity of the presented framework. 


\section{International Journal of Advanced Multidisciplinary Scientific Research (IJAMSR) ISSN:2581-4281}
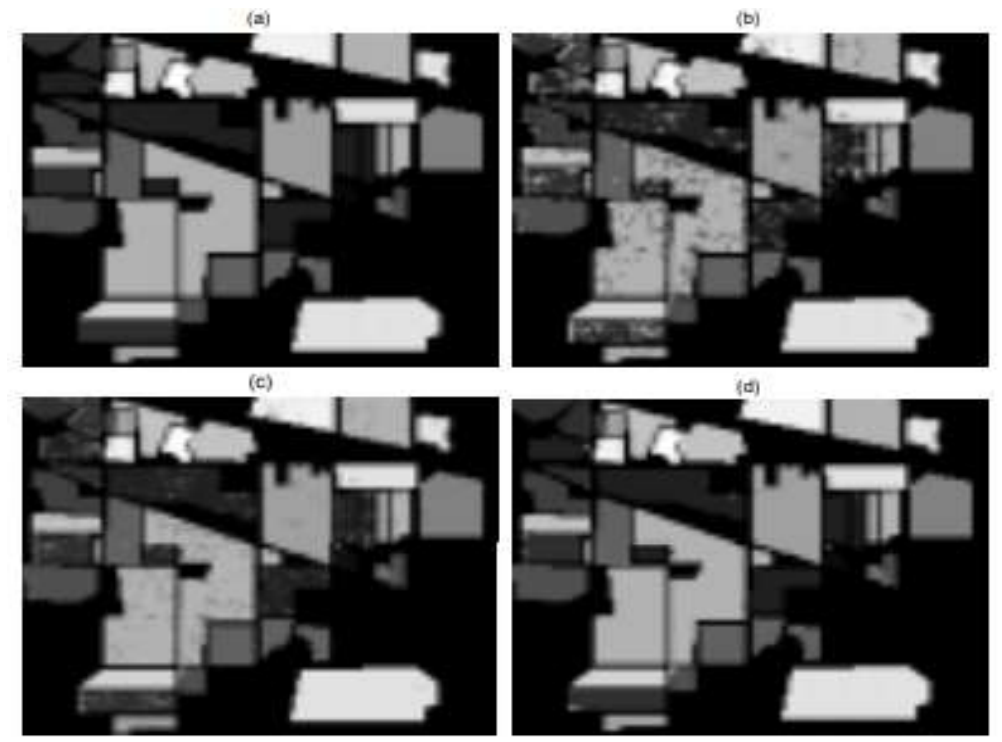

Figure 8: Classificati on results in Labeled scene and Contextual kernel window size: 5 × 5).

\section{UNL Field Hyper Supernatural Dataset}

\section{Retrieval}

The first whole-range radiometric information at $2 \mathrm{~nm}$ goal was initially broke down. To be familiar with GPR $\sigma_{b}$ band positioning, firsthey get $\sigma_{b}$ values for a solitary 10-overlap GPR simulation for LCC and gLAI arranged with all hyper phantom gatherings are. Huge distinction in the $\sigma_{b}$ qualities can be watched for the two factors. Significant is that there are not many supernatural areas with educational groups (low $\sigma_{b}$ ) furthermore, especially ineffectively educational groups (high $\sigma_{b}$ ). An unmistakable enlightening district is striking for LCC, where a deliberate area of low $\sigma_{b}$ in the red edge (around $720 \mathrm{~nm}$ ) showed up. Simultaneously, $\sigma_{b}$ of ineffectively educational groups can ascend to high qualities (here plotted in logarithmic scale), which suggests that these gatherings can be dubious in the backslide calculation. Regardless, since GPR is an information driven algorithm, and $\sigma_{b}$ relies upon what has been introduced during preparing stage, in this way, they may vacillate contingent upon the given preparing approval parceling. It bolsters the reason of utilizing the SBBR strategy in blend with a kcrease $\mathrm{CV}$ inspecting plan to construe best performing groups. Shown up at the midpoint 
IJAMSR 2 (8) www.ijamsr.com CrossRef: https://doi.org/10.31426/ijamsr.2019.2.8.1813

\section{International Journal of
I J A M S R $\begin{gathered}\text { Advanced Multidisciplinary Scientific Research (IJAMSR) ISSN:2581-4281 } \\ \text { ISA }\end{gathered}$}

of approval $R_{C V}^{2}$ results close by $\mathrm{SD}$ and minmax limits for LCC and gLAI are given. The $R_{C V}^{2}$ results of the last top 10 band mixes alongside simulation taking care of time and therelating remaining frequencies are recorded. These outcomes lead to the going with essential revelations:

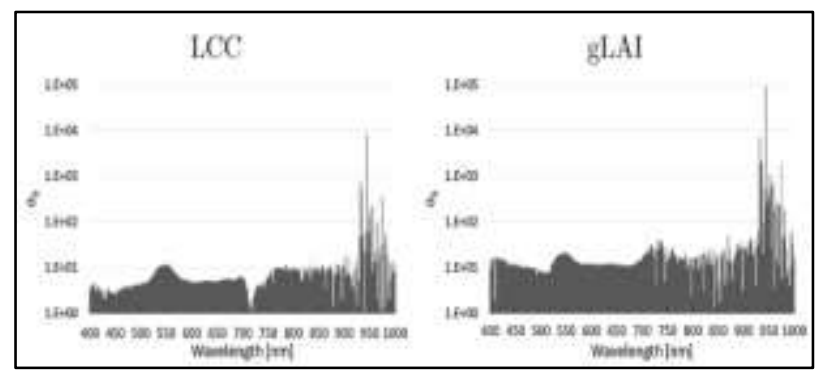

Figure 9: Obtained ${ }^{\sim}$ rankings of band.

Applying all gatherings into the GPR simulation didn't expeditious the best execution. Results were more problematic (see enormous SD, min-max) and less lucky than if there ought to be an event of for instance using only 50 to 3 best gatherings what's more, taking care of time was fundamentally greater, for instance $19 \mathrm{~s}$ for a simulation using hyper ghastly data rather than not actually a second if there ought to emerge an event of using under 10 lingering gatherings. Especially, LCC basically got from band departure, for instance presentations productively improved while having around 150 gatherings cleared.

Thusly, accuracy's stay high until three gatherings (LCC) and two gatherings (gLAI) remained. Hence, using only one band provoked most extremely horrible outcome the primary otherworldly plan that performed out and out more horrendous than while using all gatherings. 
IJAMSR 2 (8) www.ijamsr.com CrossRef: https://doi.org/10.31426/ijamsr.2019.2.8.1813

\section{International Journal of}

Advanced Multidisciplinary Scientific Research (IJAMSR) ISSN:2581-4281

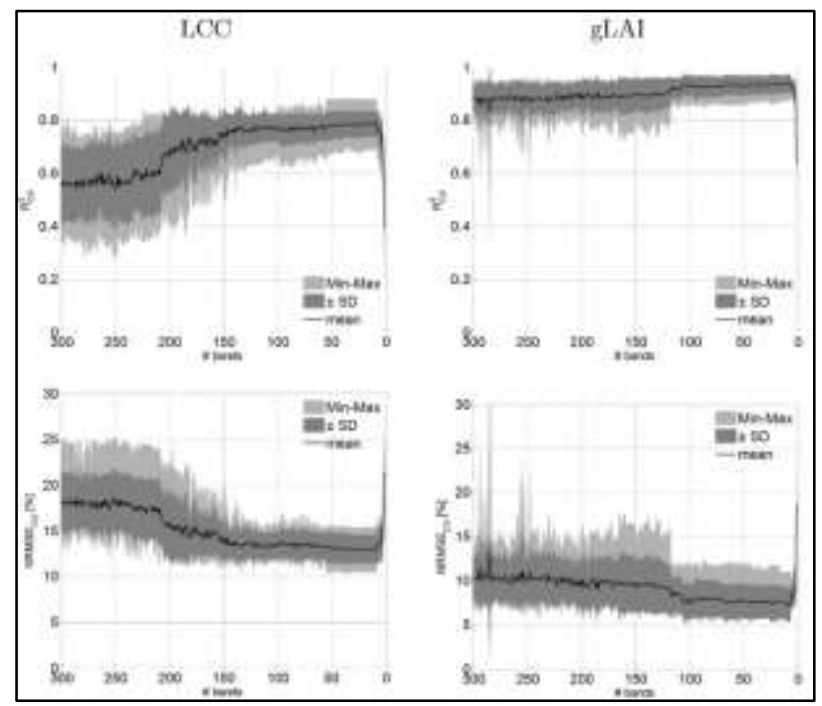

Figure 10: Cross-approval R2 CV (top) and NRMSECV [\%] (base) measurements (mean, standard deviation, and min-max ranges) for LCC (left) and gLAI (right) plotted over successively eliminating the least contributing band.

Generally, LCC benefitted most from bunches on the blue $(482 \mathrm{~nm})$, green apex $(500,564 \mathrm{~nm})$ and in the red edge $(710 \mathrm{~nm}, 714 \mathrm{~nm})$, and thusly from bunches in the NIR area $(878-980 \mathrm{~nm})$ while including more gatherings. 
IJAMSR 2 (8) www.ijamsr.com CrossRef: https://doi.org/10.31426/ijamsr.2019.2.8.1813

Table 3: Cross-validation $R_{C V}^{2}$ and NRMSECV statistics (mean, standard deviation), processing time for a single simulation and associated wavelengths for LCC and gLAI according to iterative least contributing band removal for remaining top 10 bands. Additionally, results and processing time when using all bands is provided. The best performing configuration is boldfaced.

\begin{tabular}{|c|c|c|c|c|}
\hline \# Bands & $R_{C \gamma}^{2}(S D)$ & $\operatorname{NRMSE}_{\alpha \gamma}(\mathrm{SD})$ & time (s) & Wavelengths (nm) \\
\hline \multicolumn{5}{|l|}{ LCC } \\
\hline 301 & $0.55\{0.13\}$ & $18.25(3.13)$ & 1897 & All bents \\
\hline$\vdots$ & $\vdots$ & $\vdots$ & $\vdots$ & $\vdots$ \\
\hline 10 & $0.79(0.05)$ & $1292(1.52)$ & 1.05 & $482,500,564,566,710,712,714,878,966,980$ \\
\hline 9 & $0.79(0.05)$ & $12.90(1.53)$ & 1.01 & $482,500,564,710,712,714,878,966,980$ \\
\hline 8 & $0.76(0.05)$ & $13.51(1.82)$ & 0.85 & $482,500,564,710,712,714,878,966$ \\
\hline 7 & $a 77(0.06)$ & $13.35(1.92)$ & 0.87 & $482,500,564,710,714,878,966$ \\
\hline 6 & $0.76(0.05)$ & $1351(1.75)$ & 0.83 & $482,500,710,714,878,966$ \\
\hline 5 & $0.76(0.06)$ & $13.48(1,78)$ & 0.77 & $500,710,714,878,966$ \\
\hline 4 & $073(0.06)$ & $14.10(1,74)$ & 0.69 & $500,710,714,878$ \\
\hline 3 & $0.74(0.06)$ & $14.04(1.71)$ & 0,64 & $500,710,878$ \\
\hline 2 & $0.56(0.06)$ & $18.24(2.62)$ & 0.59 & 500,710 \\
\hline 1 & $0.40(0.13)$ & $21,42(4,04)$ & 0.50 & 710 \\
\hline \multicolumn{5}{|l|}{ giAI } \\
\hline 301 & $0.88(0.12)$ & $10.07(2.48)$ & 1890 & All bands \\
\hline$\vdots$ & & : & $\vdots$ & $:$ \\
\hline 10 & $0.94(0.04)$ & $734(1.93)$ & 1.09 & $406,746,770,790,792,794,798,808,858,878$ \\
\hline 9 & $0.04(0.04)$ & $730(1.91])$ & 0.99 & $406,746,790,792,794,798,808,858,878$ \\
\hline 8 & $0.94(0.03)$ & $7.27(1.86)$ & 0.92 & $406,746,790,792,794,798,858,878$ \\
\hline 7 & $0.94(0.03)$ & $7.19(1.76)$ & 0.86 & $406,746,792,794,798,858,878$ \\
\hline 6 & $0.93(0.03)$ & $8.19(1.26)$ & 0.82 & $746,792,794,798,858,878$ \\
\hline 5 & $0.93(0.03)$ & $8.12(1.35)$ & 0.77 & $746,792,794,798,878$ \\
\hline 4 & $0.91(0.03)$ & $8.81(1.37)$ & 0.70 & $746,792,794,798$ \\
\hline 3 & $0.91(0.03)$ & $8.75(1.43)$ & 0.64 & $746,792,794$ \\
\hline 2 & $0.92(0.03)$ & $8.72(1.42)$ & 0.57 & 746,792 \\
\hline 1 & $0.64(0.11)$ & $18.77(3.49)$ & 0.51 & 792 \\
\hline
\end{tabular}

Barrax SPARC Dataset: LAI and CWC

\section{Recovery}

Next, a 4-foldCVSBBRanalysis was applied to the SPARC dataset, got at Barrax, Spain. The supernatural information starts from the mobile HyMap picture, which was organized with 125 get-togethers. This is broadly not really the field hyper supernatural dataset and causes that frightful accentuation is less an issue. That is likewise discernible in the plotted showed up at the midpoint of endorsement $\mathrm{R} 2 \mathrm{CV}$ and NRMSECV results close by SD and min-max limits. 
IJAMSR 2 (8) www.ijamsr.com CrossRef: https://doi.org/10.31426/ijamsr.2019.2.8.1813

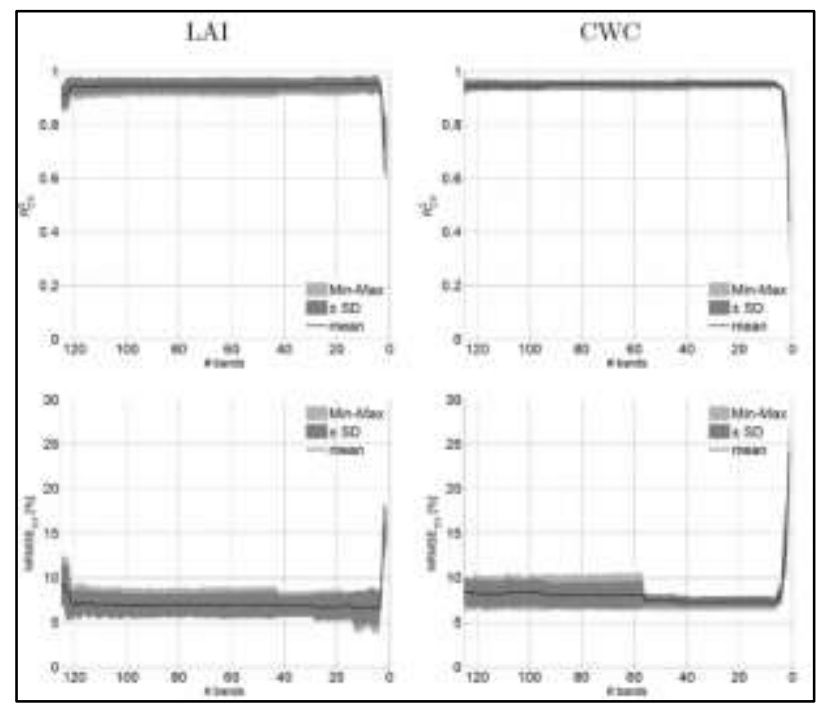

Figure 11: Cross-validation $R_{C V}^{2}$ and NRMSECV [\%] statistics.

In spite of the previous field hyper supernatural dataset, exactnesses kept stable from the fundamental 125 gatherings until only a few gatherings are left. Just, concerning the LAI dataset, it might be seen that outcomes are hazardous while using all gatherings; by reducing to 120 gatherings shows balance out. While the mean R2 CV consequences of CWC kept stable until two or three gatherings are left, while surveying the mean NRMSECV, results generally improved and kept all the more consistent when lessening to 60 gatherings until several gatherings are left. Also handling time for creating a GPR simulation improved from around $4 \mathrm{~s}$ (all gatherings) until not actually a huge part of a subsequent when confining to fewer than 10 groups. 
IJAMSR 2 (8) www.ijamsr.com CrossRef: https://doi.org/10.31426/ijamsr.2019.2.8.1813

Table 4: Cross-validation $R_{C V}^{2}$ additionally, NRMSECV estimations, taking care of time for a lone reenactment and related frequencies

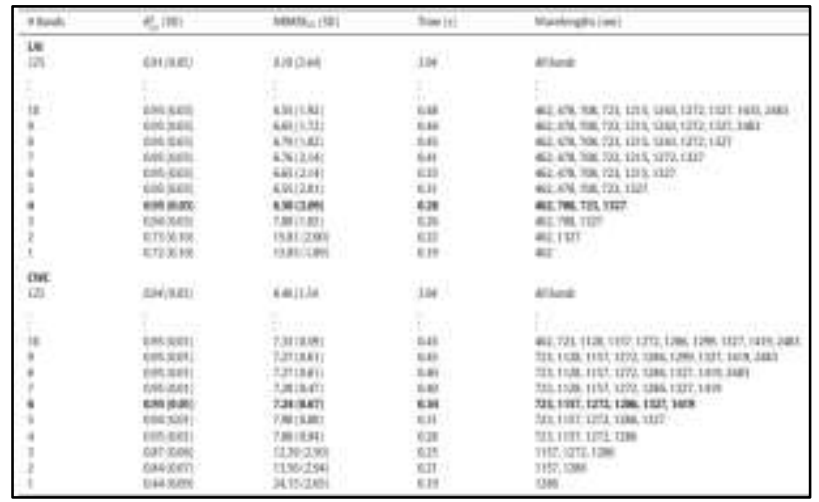

For LAI stable outcomes kept up until 4 groups are left while for CWC similar high correct nesses kept up until 6 groups are left. Along these lines, correctness is kept high until three groups (LAI) and four groups (CWC) remained. Thusly, utilizing just one band prompted most noticeably terrible result

\section{CONCLUSION}

This paper shows a few methodology of hyperspectral picture grouping utilizing Gaussian cycle, including piece level investigation of hyper otherworldly picture. With the development of hyperspectral picture mastery, hyperspectral picture gathering has been comprehensively utilized. Winning ideas strategies actually have certain limitations for more problematical hyperspectral picture grouping. Consequently, researching additionally coordinated hyperspectral picture arrangement strategies will be a critical examination course in the impending. Likewise this paper explored past works related to investigations and detecting of hyperspectral pictures are critical part of hyperspectral picture preparing utilizing Gaussian cycle. The controlled and unconfirmed gathering techniques portrayed in this paper have their relating advantages to shifting degrees. Administered arrangement involves a persuaded amount regarding certain boundaries will influence the gathering results have an effect. Accordingly, in view of disparate 
IJAMSR 2 (8) www.ijamsr.com CrossRef: https://doi.org/10.31426/ijamsr.2019.2.8.1813

\section{International Journal of \\ IJ A M S R \\ Advanced Multidisciplinary Scientific Research (IJAMSR) ISSN:2581-4281}

application necessities, joined with the procurement of hyperspectral pictures with tremendous measurements, various methodologies should be gotten together with one another so as to achieve the foreseen gathering result. Likewise effectively executed the plan of molecule swarm enhancement based gaussian cycle and breaking down hyper ghostly reflectance.

\section{REFERENCES}

1. Felipe Tobar Universidad de Chile "Bayesian Nonparametric Spectral Estimation".arXiv:1809.02196v2 [stat.ML] 12 Jan 2019.

2. Gregory R. Romanchek ,Zheng Liu,Shiva Abbaszadeh. "Kernel-based Gaussian process for anomaly detection in sparse gamma-ray data". January

23,2020https://doi.org/10.1371/journal.pone.022804 8.

3. Luca Ambrogioni Eric Maris. "Integral Transforms from Finite Data: An Application of Gaussian Process Regression to Fourier Analysis". Proceedings of the 21st International Conference on Artificial Intelligence and Statistics (AISTATS) 2018, Lanzarote, Spain. PMLR: Volume 84. Copyright 2018 by the author(s).

4. Haitao Liu, Yew-Soon Ong, Fellow, IEEE, Xiaobo Shen, and Jianfei Cai, Senior Member, IEEE. "When Gaussian Process Meets Big Data: A Review of Scalable GPs" 2019. arXiv:1807.01065v2.

5. Kai ChenEmail authorTwan van LaarhovenJinsong ChenElena Marchiori. "Incorporating Dependencies in Spectral Kernels for Gaussian Processes". Joint European Conference on Machine Learning and Knowledge Discovery in Databases ECML PKDD 2019: Machine Learning and Knowledge Discovery in Databases pp 565-581|
6. Stefano Selci. Institute for Photonics and Nanotechnologies, ARTOV C.N.R., Via del Fosso del Cavaliere 100, 00133 Roma,

7. Italy; Stefano.Selci@cnr.it"The Future of Hyperspectral Imaging". J. Imaging 2019, 5, 84; doi:10.3390/jimaging5110084 www.mdpi.com/journal/jimaging.

8. Dongyi Wang, Robert Vinson, Maxwell Holmes, Gary Seibel, Avital Bechar, Shimon Nof \& Yang Tao. "Early Detection of Tomato Spotted Wilt Virus by Hyperspectral Imaging and Outlier Removal Auxiliary Classifier Generative Adversarial Nets (OR-AC-GAN)" 2019. Open access.

9. Peng Ful, Katherine Meacham-Hensold1, Kaiyu Guan and Carl J. Bernacchil. "Hyperspectral Leaf Reflectance as Proxy for Photosynthetic Capacities: An Ensemble Approach Based on Multiple Machine Learning Algorithms". https://doi.org/10.3389/fpls.2019.00730.

10. Utsav B. Gewali.2019. "Gaussian Processes for Vegetation Parameter Estimation from Hyperspectral Data with Limited Ground Truth". https://doi.org/10.3390/rs11131614.

11. Maxim Ziatdinov, Dohyung Kim, Sabine Neumayer, Rama K. Vasudevan, Liam Collins, Stephen Jesse, Mahshid Ahmadi \& Sergei V. Kalinin. "Imaging mechanism for hyperspectral scanning probe microscopy via Gaussian process modelling. Open access. 2020.

12. Wenjing $L$ and Xiaofei Wang. "Overview of Hyperspectral Image Classification". Volume 2020 |Article ID $4817234 \quad$ | 13 pages | https://doi.org/10.1155/2020/4817234.

13. Liu Z, Abbaszadeh S, Sullivan CJ. Spatial-temporal modeling of background radiation using mobile sensor networks. PloS one. 2018 Oct 19;13(10):e0205092. pmid:30339704.

14. J. Hensman, N. Durrande, and A. Solin. Variational fourier features for Gaussian processes. Journal of Machine Learning Research, 18(151):1-52, 2018.

15.R. Boloix-Tortosa, J. J. Murillo-Fuentes, F. J. Payán-Somet, and F. Pérez-Cruz. Complex Gaussian processes for regression. IEEE Transactions on Neural Networks and Learning Systems, 29(11):5499-5511, 2018. 